standard NHS tariffs. Immunomodulators were defined as thiopurines, methotrexate or prednisolone $>20 \mathrm{mg}$ /day.

Results Between October 2008 and November 2010, 90 patients were tested with TSTB, of which 2 had a positive TSTB result and 5 borderline/indeterminate. From December 2010 until July 2012, 82 patients were tested with OFGIT, of which 3 had a positive result and 12 indeterminate/borderline (Table). 170 (99\%) had normal CXR and a negative clinical assessment. 4 of 13 patients had two sequential indeterminate IGRA and also required assessment in TB clinic. The average price per patient was $£ 60.66$ for TSTB and $£ 52.41$ for OFGIT. 88\% (152/172) have subsequently received treatment with either infliximab or adalimumab. No subjects have gone on to develop active tuberculosis.

Conclusion Using either platform, we find a comparable, low rate of LTBI in our IBD population. There appears to be a higher frequency of indeterminate results using OFGIT. This raises the average cost per patient, but overall, OFGIT remains more cost-effective than TSTB. Despite differing length of follow-up, the average time was sufficient in both, otherwise comparable, cohorts to detect likely development of active TB disease.

Abstract P57 Table 1 Characteristics of groups and results of screening assessment

\begin{tabular}{lll}
\hline & T Spot & Quantiferon \\
\hline Number of subjects & 90 & 82 \\
Median Age [years], (range) & $35(17-70)$ & 35 (17-76) \\
Risk Factors for TB & $12 \%$ & $16 \%$ \\
On immunomodulators & $63 / 90(70 \%)$ & $66 / 82$ (80\%) \\
Pos IGRA & 0 & 3 \\
Neg IGRA & 61 & 52 \\
Borderline IGRA & 1 & 2 \\
Indeterminate IGRA & 1 & 9 \\
No immodulators & $27 / 90(30 \%)$ & $16 / 82(20 \%)$ \\
Pos IGRA & 2 & 0 \\
Neg IGRA & 22 & 15 \\
Borderline IGRA & 0 & 0 \\
Indeterminate IGRA & 3 & 1 \\
Cost/assay & $£ 60.00$ & $£ 35.00$ \\
Cost/patient assessment & $£ 66.13$ & $£ 52.41$ \\
Median follow up & 29 months & 13 months \\
\hline
\end{tabular}

\section{P58 AN AUDIT OF TREATMENT OUTCOMES FOR PATIENTS WITH TUBERCULOSIS DIAGNOSED AT AN INNER LONDON TEACHING HOSPITAL BETWEEN 2000 AND 2010}

doi:10.1136/thoraxjnl-2012-202678.199

E Togun, S Stephens, 0 Sewo, FM Perrin, RD Barker. Kings College Hospital, Kings Health Partners, London, United Kingdom

Background The WHO has the ambition that $85 \%$ of patients starting treatment for TB achieve treatment success (cure or treatment complete). ${ }^{1}$ There has been considerable anxiety that poor treatment completion rates would lead to increased transmission of tuberculosis and perhaps drive increased drug resistance. We have been monitoring treatment outcomes for patients registering at our hospital since the year 2000. Here we report outcomes and analyse risk factors for treatment interruption for patients with tuberculosis registering up until 2010.

Methods All patients diagnosed with tuberculosis at an inner London teaching hospital between 2000 and 2010 were included in the study. Outcomes were recorded, as defined by the requirements of the London TB Register. Follow-up of patients is predominantly nurse-led with little day-to-day involvement from doctors. Samples are sent to the National Mycobacterial Reference Laboratory
- Whitechapel, for culture and sensitivity testing with first line anti-tuberculous drugs. Sex, Age (Decade), HIV status, disease site (pulmonary or extra pulmonary) and resistance to any first line drug were evaluated to see whether they were associated with "ost to follow up", as opposed to all other outcomes, using chi-square test for proportions.

Results One thousand two hundred and forty two patients were identified. Ten patients (1\%) had MDRTB, 714 58\% were male, 981 (79\%) were born abroad, 160 (13\%) aged 0-20 years, $679(55 \%)$ 21-40 years, 279 22\% (41-60), $12410 \%>60,164$ (13\%) were known to be HIV, 596 (48\%) had pulmonary disease 147 (18\%) of 803 with positive cultures had any drug resistance. None of the variables assessed were significantly associated with being lost to follow up.

Discussion Our nurse led TB programme has resulted in outcomes that meet international standards. Less than $5 \%$ of patients interrupt treatment. The results would probably improve if we obtained treatment outcomes for those who transferred out. None of the variables examined should be used as indicators for enhanced supervision.

1. World Health Organization - Stop TB Partnership. The Stop TB Strategy, 2006.

\section{P59 TREATMENT AND DRUG SURVEILLANCE OF LATENT TUBERCULOSIS INFECTIONS (LTBI) BY A TB PHARMACIST: A PILOT STUDY}

doi:10.1136/thoraxjnl-2012-202678.200

YO Abunga, SO Brij, G Luwemba. Peterbrough City Hospital, Peterborough, United Kingdom

Objectives To assess the safety and effectiveness of a pharmacistled LTBI clinic.

Methods Patients identified by screening as having LTBI were seen by $\mathrm{TB}$ pharmacist at the weekly TB clinic. Initial interview included history of symptoms to exclude active TB. Baseline bloods were taken as well as a screen for blood borne viruses. Follow up appointments were scheduled at 2 weeks, one month and at the end of treatment. The TB pharmacist obtains written consent for therapy, dispenses medication and information leaflets regarding potential drug adverse effects. At follow-up appointments the pharmacist evaluates treatment adherence and potential adverse effects.

Results 62 latent TB patients were seen from 01/05/11 to 01/05/12. All patients were discussed at the TB-MDT. 51 (82\%) patients were allocated to the pharmacist led clinic. The 11 (18\%) patients seen by the Consultant Respiratory Physician had significant co-morbidities at initial interview, but subsequently were followed up by the pharmacist. Of the 51 patients, 50 started therapy and 1 patient did not attend the appointment. 9 (18\%) patients reported adverse drug reaction. $46(92 \%)$ patients successfully completed treatment, 3 $(6 \%)$ patients did not complete therapy due to side effects and 1 (2\%) patient was lost to follow up. The patient who did not attend subsequently developed active TB during the study period. Of the 9 adverse drug reactions reported, only 3 required treatment to be discontinued. No adverse drug reaction occurred due to drug interaction.

Conclusion A pharmacy-led clinic for LTBI is feasible and safe. Patients were happy to be seen by the pharmacist. Patients with poly-pharmacy benefited as they had a medication review to maximise therapy and reduce adverse drug reactions.

\section{P60 HOW OFTEN DO PATIENTS WITH TUBERCULOSIS REQUIRE ENHANCED CASE MANAGEMENT?}

doi:10.1136/thoraxjnl-2012-202678.201

A Gebril, C Bell, M Woodhead. Central Manchester University Hospitals NHS Foundation Trust, Manchester, UK 
Abstract P58 Table 1 Outcomes for 1242 patients with tuberculosis diagnosed at a London teaching hospital 2000 to 2010

\begin{tabular}{|c|c|c|c|c|c|}
\hline \multicolumn{3}{|c|}{ Outcomew } & \multicolumn{3}{|c|}{ Grouped Outcome } \\
\hline & $\mathbf{n}$ & $\%$ & & $\mathbf{n}$ & $\%$ \\
\hline Completed - Non-Pulmonary & 564 & 45 & & & \\
\hline Completed - Pulmonary & 462 & 37 & & & \\
\hline $\begin{array}{l}\text { Completed - Pulmonary with } \\
\text { culture conversion }\end{array}$ & 59 & 5 & Complete & 1085 & 87 \\
\hline Died - TB cause & 10 & 1 & & & \\
\hline Died - TB contributed & 18 & 1 & & & \\
\hline Died - TB incidental & 15 & 1 & & & \\
\hline Died - TB influence unknown & 13 & 1 & Died & 56 & 5 \\
\hline Lost to follow-up & 44 & 4 & Lost to follow-up & 44 & 4 \\
\hline On treatment - initially planned & 1 & 0 & & & \\
\hline On treatment - interrupted & 1 & 0 & On treatment & 2 & 0 \\
\hline Transferred Out - LTBR ${ }^{*}$ Clinic & 40 & 3 & & & \\
\hline Transferred Out - Non LTBR Clinic & 10 & 1 & & & \\
\hline Transferred Out - Overseas & 5 & 0 & Transferred out & 55 & 4 \\
\hline Total & 1242 & 100 & & 1242 & 100 \\
\hline
\end{tabular}

An enhanced level of case management (ECM) is recommended by NICE for those deemed 'hard to reach' and should be provided where risk/needs assessment demonstrates that the patient has clinically and/or socially complex needs. NICE recommends one TB nurse for every 20 such cases so knowledge of their numbers is integral to workforce planning.

The Aim of this project was to identify and quantify those qualifying for ECM in an inner city TB cohort.

Methodology All 170 cases notified with TB in Central Manchester were retrospectively identified from department records for 01/2010 to 12/2010. Data were collected using a standard proforma from clinic letters and TB specialist nurses documentations. Standard case management was defined as per the RCN document 'Tuberculosis case management and cohort review'.

Only 60/170 (35\%) were identified as standard management cases in which $7 / 60(11 \%)$ had other co-morbidities and 4/60 (6\%) had a language barrier. 14/60 (23\%) were excluded for diverse reasons (e.g. death before diagnosis).

Results 96/170 (56\%) were identified as ECM cases which subdivided according to their requirements into either:

1. Medical needs, which comprised $12.5 \%$ (12/96) of cases including patients with dual pathology and recurrent hospital admissions.

2. Nursing needs which comprised $12.5 \%$ (12/96) of cases in which majority $(8 / 12)$ of these did not attend clinics as required and the rest $(4 / 12)$ had anti-TB treatment side effects demanding more home visits.

3. Both medical as well as nursing needs were present in $75 \%$ (72/96). In which $52 \%$ were paediatric, $11 \%$ had resistant organism, $6 \%$ were HIV +ve, $4 \%$ of patients declined treatment, $3 \%$ were on DOT, $3 \%$ had complications due to TB infection and $21 \%$ of cases had prolonged treatment due to CNS/bone involvement, interaction with other non TB treatment, immune compromised patients and overlap with others (HIV patients, Drug resistant organism, patients on DOT had prolonged treatment).

Conclusions The reasons for ECM are many and diverse and often multiple. In our practise more than half of patients could be classified as requiring such management. This has implications for TB nurse manpower planning.

\section{P61 BTS MDRTB CLINICAL ADVICE SERVICE}

doi:10.1136/thoraxjnl-2012-202678.202

'D Cullen, 2.JP Watson, ${ }^{3 P D O}$ Davies. 'British Thoracic Society, London, UK; ${ }^{2}$ Leeds General Infirmary, Leeds, UK; ${ }^{3}$ Liverpool Heart and Chest Hospital NHS Foundation Trust, Liverpool, UK

Developing an initiative begun by Professor Peter Davies and colleagues in Liverpool in 2008, the BTS has now significantly increased the functionality of this Service to offer an opportunity for genuine on line dialogue between experts and service users on all aspects of the management of patients with MDRTB.

Fully operational since July 2011-64 discrete case queries were received in the first year of operation, of which 41 were confirmed as MDRTB; 4 XDRTB; 7 Isoniazid mono-resistant and 1 related to a mycobacterium infection. The remaining 11 cases discussed were never confirmed as MDRTB or were general requests for advice.

This represents a $45 \%$ increase in case discussion since the BTS unveiled the new Service and the initial post is now regularly being followed by further requests for help as new difficulties in case management emerge.

The BTS MDRTB Clinical Advice Forum is accessible via a link on the home page of the BTS website OR directly at the url below: http://forums.brit-thoracic.org.uk/

After an on line registration is approved by the forum administrator, users are prompted to provide anonymised case details according to a pre-set questionnaire template. There is also a free text box and an opportunity to post X-ray and CT images.

\section{Lung cancer awareness, early diagnosis and staging}

\section{P62 EARLY RESULTS OF A NATIONAL LUNG CANCER AWARENESS CAMPAIGN}

doi:10.1136/thoraxjnl-2012-202678.203

J Maguire, V Kelly, M Ledson, C Smyth, A Mclver, M Walshaw. Liverpool Heart and Chest Hospital, Liverpool, United Kingdom 